\title{
Multi-Purpose Controllable Electrochemical Impedance Spectroscopy Using Synchronous Buck Converter
}

This paper was downloaded from TechRxiv (https://www.techrxiv.org).

\section{LICENSE}

$\mathrm{CCO}$

SUBMISSION DATE / POSTED DATE

$18-02-2022$ / 23-02-2022

\section{CITATION}

Abareshi, Mohammad; Sadeghi, Erfan; Saif, Mehrdad; Hamzeh, Mohsen; Alavi, Seyed Mohammad Mahdi (2022): Multi-Purpose Controllable Electrochemical Impedance Spectroscopy Using Synchronous Buck Converter. TechRxiv. Preprint. https://doi.org/10.36227/techrxiv.19140137.v1

$\mathrm{DOI}$

10.36227/techrxiv.19140137.v1 


\title{
Multi-Purpose Controllable Electrochemical Impedance Spectroscopy Using Synchronous Buck Converter
}

\author{
Mohammad Abareshi, Erfan Sadeghi, Mehrdad Saif, Mohsen Hamzeh, Seyed Mohammad Mahdi Alavi
}

\begin{abstract}
In this paper, we propose a multi-purpose controllable electrochemical impedance spectroscopy (MP-cEIS) device for online and offline battery monitoring and testing during charge and discharge processes. The proposed MP-cEIS device is based on the widely used synchronous buck converter, with a lowcost and simple input filter to provide the bidirectional operation. It also includes a closed-loop feedback system to control the injection of the EIS excitation signal. For the control system design, an average model of the proposed MP-cEIS is derived. Two methods, based on $H_{\infty}$ and quantitative feedback theory (QFT), are presented for the design of a robust controller, which guarantees the stability and tracking of the excitation signal despite model uncertainties. The excitation signal in the proposed MP-cEIS is programmable. The performance of the proposed MP-cEIS is evaluated experimentally, with multi-sine and sweptsine signals, and the results are shown.
\end{abstract}

Index Terms-Electrochemical Impedance Spectroscopy (EIS), Power Converters, Robust Control, Batteries.

\section{INTRODUCTION}

Electrochemical impedance spectroscopy (EIS) is widely used as a standard technique for battery testing, [1]. Early EIS devices are mainly suitable for laboratory tests and offline operations, [3]. With the rapid growth of electric vehicles, there is however a demand for online EIS to test the battery during its normal operations in both charge and discharge modes.

In EIS, the battery impedance spectra are calculated and analyzed by injecting an excitation signal into the battery and measuring its response. If the excitation signal is current, the voltage is measured or vice versa.

The basic idea behind the online EIS is to superimpose the excitation signal on the charge-discharge current or voltage signals, whereas, the DC part of the excitation signal is zero in the offline EIS. Based on the validity theorem, [2], the battery internal states must be stable during EIS. This condition makes the online EIS more challenging, in particular for high-power applications such as electric vehicles, where the battery is

This research was partially supported by the Natural Sciences and Engineering Research Council of Canada (NSERC)

M. Abareshi and M. Hamzeh are with the School of Electrical and Computer Engineering, University of Tehran, Tehran, Iran mohammad.abareshi.work@gmail.com,mohsenhamzeh@ut.ac.ir

E. Sadeghi and M. Saif are with the Department of Electrical and Computer Engineering, University of Windsor, Windsor, ON, Canada sadeghie@uwindsor.ca, msaif@uwindsor.ca

S. M. M. Alavi. is with the Department of Applied Computing and Engineering, School of Technologies, Cardiff Metropolitan University, Cardiff, UK.malavi@cardiffmet.ac.uk exposed to high dc currents and time-varying internal states. In such applications, a fast online EIS, within a short period, is required to ensure the internal states' stability. However, the fast EIS might cause some limitations to acquire the lowfrequency impedance spectra, which require a longer EIS.

In [3], an online EIS technique is developed, which tests the battery during the discharge mode. In the presented approach, multi-sine or noise excitation signals are generated by using a motor control system, superimposed on the discharge current. More recently, significant attempts have been made to design converter-based power electronics for online EIS. In [4], sinusoidal fluctuations are generated on the battery discharge voltage, by sinusoidal perturbations of the duty cycle of a boost converter. However, it is not clear how the duty cycle should be perturbed in order to generate a specific excitation signal such as multi-sine and swept-sine with specific amplitudes. In [5], the same structure is used for online EIS by using the step-function excitation signal, and fast Fourier transformation (FFT). The designs in [6]-[10] deal with online EIS during charging process. The design in [6] is based on a full-bridge converter, controlled in the constant-current constant-voltage charging mode to generate a superimposed swept-sine excitation signal. In [7] and [8], asynchronous and synchronous buck converters are developed for online EIS in chargers working in current control modes. The online EIS designed in [9] is based on the boost converter-based battery charger. In [10], a switched-inductor ladder converter is designed for online EIS in parallel with cell-balancing.

This brief literature review confirms that there is a lack of online switching EIS devices, with the capability to work in both discharge and charge modes without any need to change the hardware or control system. Reference [11] uses two separate unidirectional current sources to excite the battery in charging and discharging states for battery testing.

In this paper, we propose a multi-purpose controllable EIS (MP-cEIS) device for online and offline applications, with the capability to switch between the discharge and charge modes without any need to change the hardware or control system. The novel contributions of this work are summarized as follows:

- By adding a low-cost and simple input filter, the widely used synchronous buck converter is developed to support the bidirectional operation in MP-cEIS.

- The average model of the proposed MP-cEIS is developed for the control system design. 


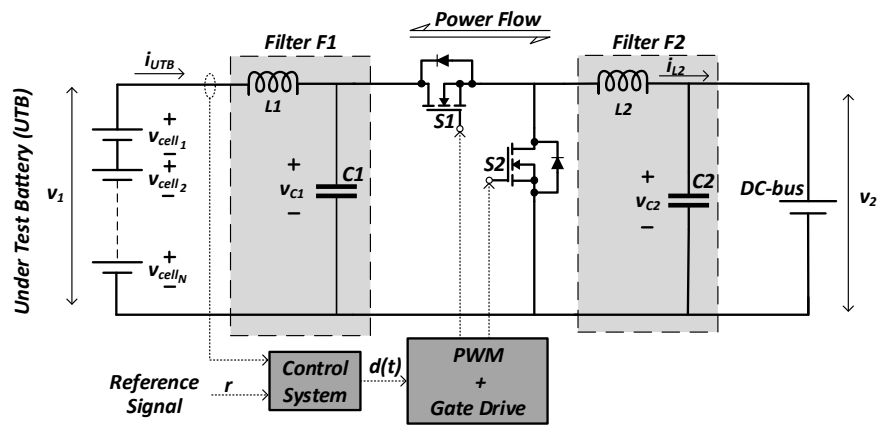

Fig. 1. Schematic of the bidirectional converter, utilized for bidirectional and controllable EIS (bc-EIS). The positive direction of $i_{U T B}$ and $i_{L 2}$ is shown.

- Two design methods based on $H_{\infty}$ and quantitative feedback theory (QFT), are described for the robust control of the excitation signal despite model uncertainties. In [8], the QFT-based design was just explained.

- The performance of the proposed MP-cEIS is evaluated experimentally, with multi-sine and swept-sine signals, and the results are shown.

The rest of the paper is organized as follows. In section II, the schematic, operation, and modeling of the proposed bc-EIS circuit are described. In section III, the design of the QFT and $H_{\infty}$ robust control systems are explained. Finally, practical implementation and results are presented in sectionIV.

\section{Power Electronics: Schematic, Operation, And AVERAGE Model}

Figure 1 shows the proposed power electronics, which is based on the well-known synchronous buck converter. The novelty is to add the filter $F_{1}$ to make $i_{U T B}$ a continuous signal with respect to time for bidirectional EIS. Overall, the MP-cEIS consists of two LC filters $F_{1}$ and $F_{2}$, and two semiconductor switches, $S_{1}$, and $S_{2}$, which control the direction of the current flow. The under-test battery (UTB) is connected to the input of $F_{1}$. A DC bus, by using supercapacitors [12] or batteries [13], is connected to the output of $F_{2}$, with $V_{2}<V_{1}$.

The overall operation of the presented MP-cEIS is as follows. First, a reference current signal $r(t)$ is defined. As elaborated in [14] and [8], the selection of $r(t)$ should be chosen based on the concepts of persistent expiation and system identification.

The responsibility of the robust control system is to switch $S_{1}$ and $S_{2}$ on and off by an appropriate duty cycle $d$, such that the current $i_{U T B}$ satisfactorily tracks the reference signal $r$ in the presence of uncertainties. It should be noted that the gate signal $S_{1}$ is toggled of the gate signal $S_{2}$. In addition, the desired and actual currents $r(t)$ and $i_{U T B}$ are positive when UBT is tested during the discharge process, and are negative during charge. During the offline test, the offset (i.e., mean) of $r(t)$ and $i_{U T B}$ is zero.

Figure 1 shows the MP-cEIS power electronics for a battery module with series cells. The impedance spectra of the $i$-th battery cell are computed by using $i_{U T B}$ and $V_{\text {cell }_{i}}$. The computation of the impedance spectra was elaborated in literature [3], [8], [14], [15]. By using the same bidirectional converter, a programmable MP-cEIS device could also be designed for battery packs consisting of parallel modules, to test all modules simultaneously, or individually, with the same or different excitation signals. It should be noted that fundamentals of the control system design, discussed in section III, also remain unchanged in the development of the MP-cEIS to battery packs applications.

By using the well-known averaging method [16], a smallsignal state-space model of the bidirectional converter is given by:

$$
\begin{aligned}
& \dot{\hat{x}}(t)=A x(t)+B u(t)+B_{d} d(t), \\
& y(t)=H x(t)
\end{aligned}
$$

where, $x, y, d, \quad$ and $u$ are small-signals of $\left[\begin{array}{llll}i_{U T B} & i_{L_{2}} & v_{C_{1}} & v_{C_{2}}(t)\end{array}\right]^{T}, \quad i_{U T B}$, duty cycle, and $\left[\begin{array}{ll}v_{1} & v_{2}\end{array}\right]^{T}$, respectively, and

$$
\begin{aligned}
& A=\left[\begin{array}{cccc}
-\frac{R_{b}}{L_{1}} & 0 & -\frac{1}{L_{1}} & 0 \\
0 & -\frac{R_{o n}}{L_{2}} & \frac{D}{L_{2}} & -\frac{1}{L_{2}} \\
\frac{1}{C_{1}} & -\frac{D}{C_{1}} & 0 & 0 \\
0 & \frac{1}{C_{2}} & 0 & -\frac{1}{C_{2} R_{s}}
\end{array}\right], B=\left[\begin{array}{cc}
\frac{1}{L_{1}} & 0 \\
0 & 0 \\
0 & 0 \\
0 & \frac{1}{C_{2} R_{s}}
\end{array}\right] \\
& B_{d}=\left[\begin{array}{lllll}
0 & 0 & \frac{V_{2}-V_{1}}{C_{1}\left(R_{s}+R_{o n}+D R_{b}\right)} & 0
\end{array}\right]^{T}, H=\left[\begin{array}{cccc}
1 & 0 & 0 & 0
\end{array}\right]
\end{aligned}
$$

where, $D$ is the duty cycle at steady state, $R_{o n}$ is internal resistance of the switches, $R_{s}$ is internal resistances of the DC bus, and $R_{b}$ is internal resistance of battery cells.

The plant's transfer function from $d$ to $i_{U T B}$ is obtained as follows:

$$
P(s)=H(s I-A)^{-1} B_{d}
$$

where, $s$ is the Laplace operator.

\section{QFT And $H_{\infty}$ Robust Control Systems Design}

Consider the standard control system Figure 2. In this control structure, the current of UTB, $i_{U T B}$ is measured and compared with the reference signal $r(t)$. The difference is fed into the controller $G(s)$. The objective is to design a robust controller $G(s)$, and generate a desired duty cycle for the semiconductor switches $S_{1}$ and $S_{2}$, such that, the current $i_{U T B}(t)$ satisfactorily tracks the reference signal $r(t)$ with respect to the existing uncertainties $\mathcal{P}$, while the stability of the closed-loop system is also preserved. In this section, we propose two methods for the design of the robust controller $G(s)$ based on $H_{\infty}$ and QFT theories. In both methods, the robust stability and performance constraints are firstly defined. In QFT, these constraints result in some bounds in Nichols chart [17], [18]. The controller is then designed by shaping the system frequency response to satisfy these bounds. In $H_{\infty}$, the controller is obtained through various computational algorithms; algebraic Riccati equations, semidefinite programming, linear and bilinear matrix inequalities (LMIs and BMIs), nonsmooth optimization, etc., see [19] and reference therein. There are Matlab toolboxes for both QFT [20], [21] and $H_{\infty}$ [22].

In the following, the QFT and $H_{\infty}$ design methods are briefly explained for robust control of the excitation current 


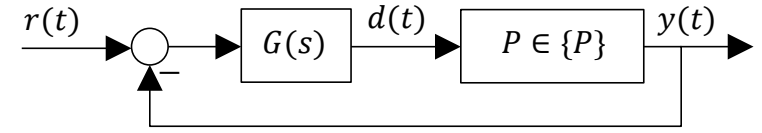

Fig. 2. Structure of the Robust Control System.

in MP-cEIS, with the circuit values given in section IV, and uncertainties on the internal resistances of the battery cells and DC-bus between 1 to $200 \mathrm{~m} \Omega$.

\section{A. QFT Robust Control}

The following constraints are needed for the design of the QFT-based robust controller.

$$
\begin{gathered}
\quad\left|\frac{P G}{1+P G}(j \omega)\right| \leq \gamma \\
\quad \text { for all } P \in \mathcal{P} \text {, and } \omega \in\left\{\omega_{\text {stability }}\right\} \\
\left|M_{l}(j \omega)\right| \leq\left|F \frac{G P}{1+G P}(j \omega)\right| \leq\left|M_{u}(j \omega)\right| \\
\text { for all } P \in \mathcal{P} \text {, and } \omega \in\left\{\omega_{\text {tracking }}\right\}
\end{gathered}
$$

The constraint (4) guarantees the robust stability by providing a desired gain and phase margins over the uncertainty region $\mathcal{P}$, and desired frequencies $\omega_{\text {stability. The constraint (5) }}$ deals with the tracking performance, which pushes the closedloop system response to lie between a lower band, $M_{l}$, and an upper band, $M_{u}$ over the frequency range of $\omega_{\text {tracking. }}$. The selection of $\gamma, \omega_{\text {stability }}, M_{l}, M_{u}$, and $\omega_{\text {tracking }}$ was elaborated in [8]. In this work, we choose $\gamma=1.2$ and $\omega_{\text {stability }}=\left\{10^{6}, 10^{7}\right\} \mathrm{rad} / \mathrm{sec}$ which implies G.M. $\approx 1.83$ and P.M. $\approx 49.25^{\circ}$. We also choose

$$
\begin{aligned}
& M_{l}(s)=\frac{1}{\left(\frac{1}{10000^{2}} s^{2}+\frac{2}{10000} s+1\right)\left(\frac{1}{7500} s+1\right)\left(\frac{1}{5000} s+1\right)^{2}} \\
& M_{u}(s)=\frac{1}{\left(\frac{1}{7500^{2}} s^{2}+\frac{1}{7500} s+1\right)}
\end{aligned}
$$

which, guarantee the maximum overshoot of around $15 \%$ and settling time of the closed-loop response to be around $2 \mathrm{msec}$. We compute the tracking performance for frequencies below the desired bandwidth, $\omega_{\text {tracking }}=\left\{1,10,100,10^{3}\right\} \mathrm{rad} / \mathrm{sec}$, where the magnitude of $M_{l}$ and $M_{u}$ is $0 \mathrm{~dB}$. In such a case, the is no need for the pre-filter, which is usually used in the QFT robust control systems. In order to guarantee zero steadystate error, a controller is designed with appropriate integral terms.

The solutions of (4) and (5) divide the Nichols chart into acceptable and unacceptable regions at each design frequency. The feedback compensator $G$ is shaped such that the loopfunction $L_{f}(s)=P_{o} G(s)$ lies within the acceptable region for a given nominal plant $P_{o}$. Fig. 3 shows the design bounds in the Nichols chart, computed by using the QFT toolbox [21]. It is seen that the loop-function satisfies all design bounds by using the following integral controller

$$
G_{Q F T}(s)=\frac{150}{s} \text {. }
$$

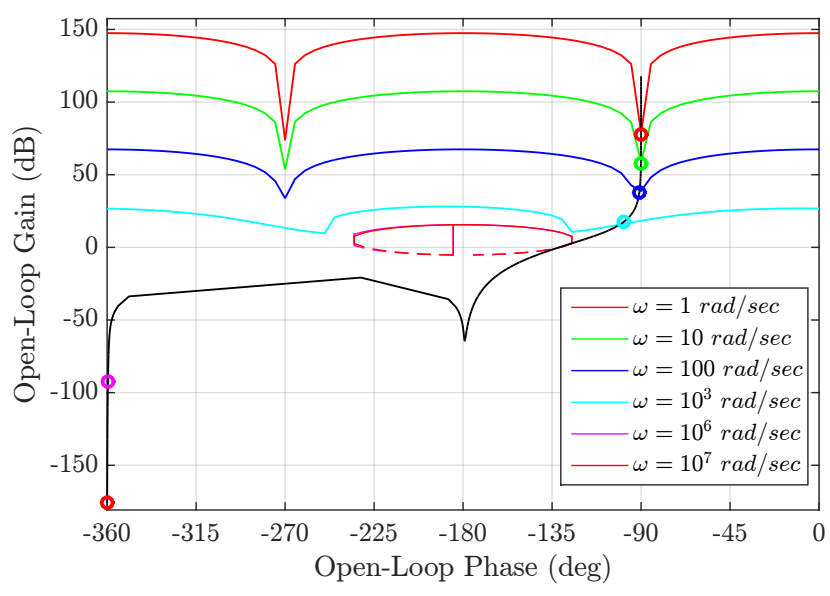

Fig. 3. The bounds for the design of the feedback compensator, and the loop-function, which satisfies all bounds. The loop-function at each design frequency lies above the corresponding solid lines, and below the dashed lines. The loop-function does not enter the high-frequency stability contours.

\section{B. $H_{\infty}$ Robust Control}

In $H_{\infty}$ theory, the controller is obtained by solving the following design constraint, [19]

$$
\left\|\begin{array}{l}
W_{T} T \\
W_{S} S
\end{array}\right\|_{\infty}<1
$$

where, $S$ and $T$ are the sensitivity and complementary sensitivity functions, respectively. $W_{T}$ and $W_{S}$ are the corresponding weighting functions. For tracking performance, $W_{T}$ is chosen such that the gain of its inverse is unity below the bandwidth. Above the bandwidth, the gain of $W_{T}^{-1}$ decreases with a desired rate. For consistency with the QFT design, we choose $W_{T}=M_{u}^{-1}$. The weighting function $W_{S}$ is complementary to $W_{T}$, i.e., its gain is small below the bandwidth, and becomes large above that. In this work, a proper $W_{S}$ is given by:

$$
W_{S}=\frac{0.8333 s+4 \times 10^{5}}{s+4000} \text {. }
$$

For consistency with the QFT design, an integral controller is designed by using the fixed-structure $H_{\infty}$ design method [19]. The $H_{\infty}$ problem (7) is computationally solved by using generalized gradients and bundling techniques and Matlab's hinfstruct command, which results in the following integral controller

$$
G_{H_{\infty}}(s)=\frac{41.2}{s} .
$$

\section{Practical Implementation and Results}

A photo of the fabricated MP-cEIS is shown in Figure 4. Two series lithium-ion battery cells are tested. Another lithium-ion battery is used as the DC-bus. Components of the bidirectional converter is chosen as: $L_{1}=3.3 \mu \mathrm{H}, L_{2}=$ 


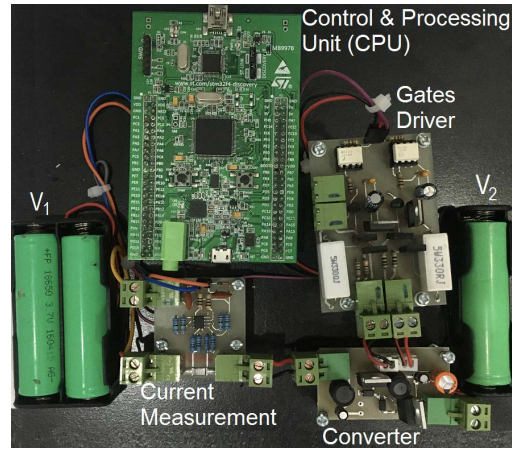

Fig. 4. Photograph of the experimental setup.

$11.2 \mu H, C_{1}=1 \mu F, C_{2}=100 \mu F$. The switching frequency is set to $200 \mathrm{kHz}$. The STMicroelectronics' microcontroller STM32F407VGT6 is used for the digital implementation of the designed robust controllers (6) and (9). The Tustin transform is used for obtaining the discrete format of the controllers. The control signal is updated with the rate of $200 k H z$. The current signal $i_{U T B}$ is sampled by the sampling frequency $f_{\text {sampling }}=200 \mathrm{kHz}$.

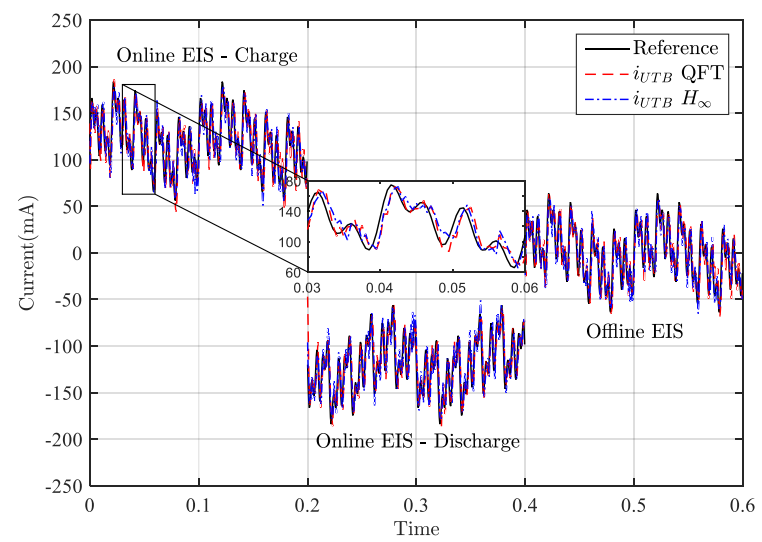

(a) Multi-sine excitation current

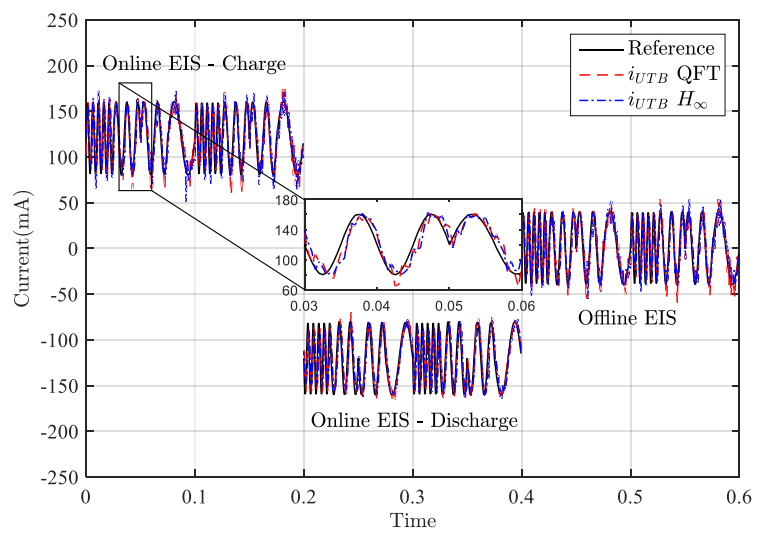

(b) Swept-sine excitation current

Fig. 5. Tracking of multi-sine and swept-sine excitation currents during online and offline modes using the proposed QFT and $H_{\infty}$ controllers.

It is shown in Fig. 5 that the excitation current generated and controlled by the MP-cEIS satisfactorily tracks the desired reference signals. Without loss of generality, two widely used

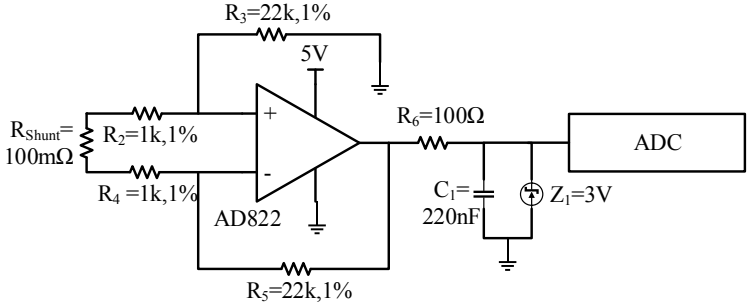

Fig. 6. Schematic of current measuring circuit.

reference signals are tested: multi-sine and swept-sine signals. The reference multi-sine signal is the sum of four sinusoidal signals with the equal amplitudes of $20 \mathrm{~mA}$, and frequencies $10,50,100,200 \mathrm{~Hz}$. The amplitude of the swept-sine signal is $40 \mathrm{~mA}$ with the frequency varying between 40 and $200 \mathrm{~Hz}$. Both signals are superimposed on $+120 \mathrm{~mA}$ and $-120 \mathrm{~mA}$ DC during discharge and charge processes, respectively. Figure 5 shows the performance of the MP-cEIS with the proposed QFT and $H_{\infty}$ controllers (6) and (9). It is seen that $i_{U T B}$ generated and controlled by the MP-cEIS satisfactorily tracks the desired reference signals with both QFT and $H_{\infty}$ controllers.

As shown in Fig. 6, the high-side current-sensing method was used to measure the current accurately. In this method, which uses a shunt resistor, the voltage drop across the shunt resistor $\left(R_{\text {shunt }}=0.1 \Omega\right)$ is amplified (22 times here) and given to the ADC microcontroller unit. The full transfer function of the circuit is provided below.

$$
V_{o}=I_{\text {in }} R_{\text {shunt }} \frac{R_{5}}{R_{4}}
$$

In order to calculate the maximum shunt resistance, the maximum voltage across the shunt is considered $100 \mathrm{mV}$.

$$
R_{\text {shunt }}=\frac{V_{\max }}{I_{\max }}=\frac{100 \mathrm{mV}}{1 \mathrm{~A}}=100 \mathrm{~m} \Omega
$$

The gain of the amplifier is set to maximize the output swing range.

$$
\text { Gain }=\frac{V_{\text {Omax }}-V_{\text {Omin }}}{\left(I_{\text {imax }}-I_{\text {imin }}\right) R_{\text {shunt }}}
$$

\section{Conclusions}

We proposed a buck-converter-based power electronics for multi-purpose controllable electrochemical impedance spectroscopy (MP-cEIS) for online and offline battery monitoring and testing during charge and discharge processes. By adding a filter, controllable continuous currents are generated at both sides of the buck converter for bidirectional EIS. We designed and implemented two robust control systems for the presented MP-cEIS, by using QFT and $H_{\infty}$, considering battery model uncertainties. The effectiveness of the proposed MP-cEIS was tested experimentally on a battery module with series cells, and we also discussed about the extension to battery packs applications.

\section{REFERENCES}

[1] D. A. Howey, S. M. M. Alavi, "Rechargeable battery energy storage system design," Handbook of Clean Energy Systems, Vol. 5, pp. 28012818, John Wiley \& Sons, Ltd., 2015. 
[2] Yuan, X. Z., Song, C., Wang, H., and Zhang, J., "Electrochemical impedance spectroscopy in PEM fuel cells: fundamentals and applications", Springer, 2010.

[3] D. A. Howey, P. D. Mitcheson, V. Yufit, G. J. Offer, N. P. Brandon, "Online measurement of battery impedance using motor controller excitation," IEEE Trans. on Vehicular Technology, 63 (6), pp. 2557-2566, 2014.

[4] W. Huang and J. A. Abu Qahouq, "An online battery impedance measurement method using DC-DC power converter control," IEEE Trans. on Industrial Electronics, Vol. 61, No. 11, pp. 5987 - 5995, 2014.

[5] J. A. Abu Qahouq and Z. Xia, "Single-Perturbation-Cycle Online Battery Impedance Spectrum Measurement Method with Closed-Loop Control of Power Converter,' IEEE Trans. Industrial Electronics, Vol. 64, No. 9, pp. 1-1, 2017.

[6] Y.-D. Lee, S.-Y. Park, and S.-B. Han, "Online embedded impedance measurement using high-power battery charger," IEEE Trans. on Industry Applications, Vol. 51, No. 1, pp. 498 - 508, 2015.

[7] S M R. Islam, S.-Y. Park, "Precise On-line Electrochemical Impedance Spectroscopy Strategies for Li-Ion Batteries," IEEE Trans. on Industry Applications, Vol. 56, No. 2, pp. 1661 - 1669, 2019.

[8] E. Sadeghi, M. H. Zand, M. Hamzeh, M. Saif, S. M. M. Alavi, "A Controllable Electrochemical Impedance Spectroscopy: From Circuit Design to Control and Data Analysis" IEEE Trans. on Power Electronics, 35(9), pp. 9935-9944, 2020.

[9] T. N. Gucin, L. Ovacik, "Online Impedance Measurement of Batteries Using the Cross-Correlation Technique," IEEE Trans. on Power Electronics, 35(4), pp. 4365-4375, 2020.

[10] E. Din, C. Schaef, K. Moffat, J. T. Stauth, "A Scalable Active Battery Management System With Embedded Real-Time Electrochemical Impedance Spectroscopy," IEEE Trans. on Power Electronics, 32(7), pp. 5688-5698, 2017.

[11] P. Wekamp, P. Haumann and J. Melbert, "600-A Test System for Aging Analysis of Automotive Li-Ion Cells With High Resolution and Wide Bandwidth," in IEEE Transactions on Instrumentation and Measurement, vol. 65, no. 7, pp. 1651-1660, July 2016.

[12] K. Bi, L. Sun, Q. An and J. Duan, "Active SOC Balancing Control Strategy for Modular Multilevel Super Capacitor Energy Storage System,' in IEEE Transactions on Power Electronics, vol. 34, no. 5, pp. 4981-4992, May 2019.

[13] S. Chowdhury, M. N. B. Shaheed and Y. Sozer, "State-of-Charge Balancing Control for Modular Battery System With Output DC Bus Regulation," in IEEE Transactions on Transportation Electrification, vol. 7, no. 4, pp. 2181-2193, Dec. 2021.

[14] S. M. M. Alavi, A. Mahdi, S. J. Payne, D. A. Howey, "Identifiability of generalised Randles circuit models," IEEE Trans. on Control Systems Technology, 25 (6), 2112-2120, 2017.

[15] P. E. Jacob, S. M. M. Alavi, A. Mahdi, S. J. Payne, D. A. Howey, "Bayesian Inference in Non-Markovian State-Space Models With Applications to Battery Fractional-Order Systems," IEEE Trans. on Control Systems Technology, 26 (2), pp. 497-506, 2018.

[16] R. D. Middlebrook, S. Cuk, "A general unified approach to modelling switching-converter power stages," IEEE Power Electronics Specialists Conference, pp. 73 - 86, 1976.

[17] C. H. Houpis, S. J. Rasmussen, M. Garcia-Sanz, "Quantitative Feedback Theory: Fundamentals and Applications, 2nd Edition, CRC Press, December 2005.

[18] SMM Alavi, MJ Hayes, "Quantitative feedback design for a benchmark quadruple-tank process," IET Irish Signals and Systems Conference, 401406, 2006.

[19] P. Apkarian and D. Noll, "Nonsmooth $H_{\infty}$ Synthesis," IEEE Trans. on Automatic Control, Vol. 51, No. 1, pp. 71 - 86, 2006.

[20] M. Garcia-Sanz, "The QFT Control Toolbox (QFTCT) for Matlab," 2008.

[21] C. Borghesani, Y. Chait, O. Yaniv, "Quantitative Feedback Theory Toolbox Users Guide, Terasoft Inc., 1993-2003.

[22] G. Balas, R. Chiang, A. Packard, M. Safonov, "Robust Control Toolbox Reference," The MathWorks, Inc., 2020. 